\title{
Topotecan Monotherapy in Heavily Pretreated Patients with Progressive Advanced Stage Neuroendocrine Carcinomas
}

\author{
Ingrid Holst Olsen 1,2,4,5, ${ }^{\bowtie}$, Ulrich Knigge2,5,6, Birgitte Federspiel3,6, Carsten Palnæs Hansen2,6, Anna Skov², \\ Andreas Kjær ${ }^{4,5,6}$ and Seppo W. Langer ${ }^{1,6}$ \\ 1. Depts of Oncology 5073, Rigshospitalet, Faculty of Health Sciences, University of Copenhagen, Denmark; \\ 2. Depts of Surgery 2122, Rigshospitalet, Faculty of Health Sciences, University of Copenhagen, Denmark; \\ 3. Depts of Pathology 5442, Rigshospitalet, Faculty of Health Sciences, University of Copenhagen, Denmark; \\ 4. Depts of Clinical Physiology, Nuclear Medicine \& PET 4011, Rigshospitalet, Faculty of Health Sciences, University of Copenhagen, \\ Denmark; \\ 5. Cluster of Molecular Imaging, Rigshospitalet, Faculty of Health Sciences, University of Copenhagen, Denmark; \\ 6. European NET Centre of Excellence, Rigshospitalet, Faculty of Health Sciences, University of Copenhagen, Denmark.
}

$\square$ Corresponding author: Ingrid Holst Olsen, MD. Dept. of Surgery C 2122, Copenhagen University Hospital, Rigshospitalet, Blegdamsvej 9, DK-2100 Copenhagen, Denmark. Email: ingrid.marie.holst.olsen@rh.regionh.dk Fax: +45 35327546.

( ) Ivyspring International Publisher. This is an open-access article distributed under the terms of the Creative Commons License (http://creativecommons.org/ licenses/by-nc-nd/3.0/). Reproduction is permitted for personal, noncommercial use, provided that the article is in whole, unmodified, and properly cited.

Received: 2014.04.16; Accepted: 2014.07.02; Published: 2014.08.01

\begin{abstract}
Background: Neuroendocrine carcinomas (WHO grade 3) are highly aggressive tumors with an immense tendency to metastasize and with a poor prognosis. In advanced disease, there is no standard treatment beyond first-line platin/etoposide-based chemotherapy. Topotecan is widely used as second-line treatment in small cell lung cancer, which also responds markedly on first-line platin/etoposide. Hence, we investigated the feasibility of topotecan in previously treated patients with neuroendocrine carcinomas.

Material and Methods: Retrospective analysis of 22 patients with disseminated and progressive neuroendocrine carcinomas (Ki67>20\%, G3) successively treated with oral topotecan $2.3 \mathrm{mg} / \mathrm{m}^{2}$ dI-5 every 3 weeks. All patients had previously received treatment with carboplatin/etoposide. Demographic, clinical and pathological features were recorded. CT-evaluations according to RECIST I.I were performed after every three courses. Hematological toxicity was assessed by CTC-criteria.

Results: Twenty-two eligible patients received a median of 2 courses [range I-6]. Median age: 65 years [35-77]. Male/female: II/II. Median Ki-67 index: 95\% [25-100\%]. Median number previous chemotherapy regimens: 2 [I-3]. All patients were evaluable for response: Five achieved stable disease (SD) and 17 progressed (PD). The median overall survival for the 22 patients was 3.2 months and the median progression-free survival was 2.1 months. The one-year survival was $18 \%$. There were no treatment related deaths. The treatment was well tolerated: Haematological toxicity comprised leukopenia CTC grade 3 (14\%), grade $4(9 \%)$ and thrombocytopenia grade 3 (14\%).

Conclusion: Topotecan monotherapy shows modest anti-tumor activity in heavily treated patients with progressive disseminated $\mathrm{G} 3$ neuroendocrine carcinomas.
\end{abstract}

Key words: neuroendocrine carcinomas, chemotherapy, topotecan. 


\section{Introduction}

Neuroendocrine carcinomas (NECs) accounts for more than $10 \%$ of gastrointestinal neuroendocrine tumors [1]. NECs are highly malignant and the prognosis is often very poor despite treatment.

The optimal treatment strategy is challenging. First-line treatment has largely remained unchanged since the study by Moertel et al. in the early 1990s, which highlighted the efficacy and feasibility of using a chemotherapy treatment regimen for patients with small cell lung cancer (SCLC) in patients with NECs [2]. Beyond this first-line treatment there is no further well-documented standard regimen of treatment. Validated prognostic factors are needed to predict the aggressiveness of the tumor and thereby the optimal treatment for each patient.

Treatment with topotecan is associated with prolongation of survival and quality of life benefit in patients with relapsed SCLC [3]. Despite the lack of published studies, topotecan treatment has been transferred into the guidelines of The North American Neuroendocrine Tumor Society (NANETS) and the National Comprehensive Cancer Network (NCCN) as an option in patients with advanced NECs in relapsed patients $[4,5]$.

Topotecan is a semisynthetic water-soluble derivative of camptothecin (CPT) and inhibits the cut-and-repair nuclear enzyme DNA topoisomerase I. It is predominantly used to treat SCLC, ovarian and cervical cancer, neuroblastoma, and glioma. Furthermore, topotecan has demonstrated significant activity as second-line therapy in recurrent SCLC both as intravenous and as oral formulation [6].

We present a retrospective analysis of patient characteristics and treatment efficacy in heavily pretreated patients with advanced stage NEC who received treatment with topotecan during 3 years period at the Department of Oncology, European NET Centre of Excellence, Rigshospitalet, Copenhagen, Denmark.

\section{Material and Methods}

\section{Patients and Treatment}

We retrospectively evaluated 22 patients with disseminated NEC (Ki-67>20\%) and disease progression after previous exposure to carboplatin and etoposide and eligible for topotecan treatment from January 2010 to March 2013. All patients had adequate hematological, renal and hepatic function and WHO performance status $0-4$. Topotecan was administered orally for 5 consecutive days every 3 weeks and patients received a dose of $2.3 \mathrm{mg} / \mathrm{m}^{2}$. Radiological tumor response was classified according to Response Evaluation Criteria in Solid Tumors (RECIST 1.0)
Patient characteristics are summarized in Table 1. No patients were lost to follow up.

Table I. Patient Characteristics.

\begin{tabular}{|c|c|c|}
\hline & Valid Cases & $\mathrm{N}(\%)$ \\
\hline No of patients & 22 & \\
\hline Male:Female & $11: 11$ & $50: 50$ \\
\hline Median Age, y, (Range) & $65(35-77)$ & \\
\hline \multicolumn{3}{|l|}{ Performance Status WHO } \\
\hline $0,1,2,3,4$ & $\begin{array}{l}6 / 10 / 3 / 2 \\
/ 1\end{array}$ & $\begin{array}{l}27.0 / 45.5 / \\
14.0 / 9.0 / 4.5\end{array}$ \\
\hline \multicolumn{3}{|l|}{ Primary Tumor } \\
\hline Pancreas & 2 & 9 \\
\hline Esophagus/Cardia & 8 & 36 \\
\hline Small Intestine & 2 & 9 \\
\hline Colon & 1 & 5 \\
\hline CUP (Cancer of unknown primary) & 9 & 41 \\
\hline \multicolumn{3}{|l|}{ Chromogranin A Immunohistochemistry } \\
\hline $\begin{array}{l}\text { Strongly positive / Positive/ Weakly posi- } \\
\text { tive/ Negative / Not Done }\end{array}$ & $\begin{array}{l}4 / 8 / 7 / 1 / \\
2\end{array}$ & $\begin{array}{l}18 / 36 / 32 / \\
5 / 9\end{array}$ \\
\hline \multicolumn{3}{|l|}{ Synaptophysin Immunohistochemistry } \\
\hline $\begin{array}{l}\text { Strongly positive / Positive/ Weakly posi- } \\
\text { tive/ Negative }\end{array}$ & $11 / 9 / 1 / 1$ & $\begin{array}{l}50.0 / 41.0 / \\
4.5 / 4.5\end{array}$ \\
\hline \multicolumn{3}{|l|}{ Somatostatin Receptor Scintigraphy } \\
\hline Strongly positive (>liver uptake) & 3 & 14.5 \\
\hline Weakly Positive (<liver uptake) & 3 & 14.5 \\
\hline Negative & 10 & 46.0 \\
\hline Not done & 6 & 27.0 \\
\hline \multicolumn{3}{|l|}{ Ki-67 } \\
\hline Median, \%, (Range) & $95(25-100)$ & \\
\hline$\geq 55 \%$ & 17 & 77 \\
\hline$<55 \%$ & 5 & 23 \\
\hline \multicolumn{3}{|l|}{ Site of metastases } \\
\hline Liver & 11 & \\
\hline Lymph nodes & 19 & \\
\hline Brain & 3 & \\
\hline Bone & 5 & \\
\hline Pleural & 1 & \\
\hline Lung & 3 & \\
\hline Skin & 2 & \\
\hline Breast & 1 & \\
\hline Peritoneal, Retroperitoneal & 4 & \\
\hline \multicolumn{3}{|l|}{ No. of metastatic sites } \\
\hline 1 & 7 & 32 \\
\hline 2 & 7 & 32 \\
\hline$\geq 3$ & 8 & 36 \\
\hline
\end{tabular}

\section{Study type and ethical considerations}

Topotecan appears in international guidelines as a treatment option in NEC, and as such is an established but non-validated standard treatment. The study is a retrospective quality assurance of the treatment efficacy that was approved and supervised by the Institutional Study Review Board, and follows the requirements for maintenance of the institutions certification as a Center of Excellence accredited by the European Neuroendocrine Tumor Society (ENETS). Data handling was handled in a non-personalized matter (patient-numbering). All patients had given signed informed consent to the treatment. 


\section{Immunohistochemistry (IHC)}

Formalin-fixed paraffin-embedded tissue samples were cut at $4 \mu \mathrm{m}$ thick sections and mounted on coated slides (Dako Flex IHC microscope slides). To remove the paraffin EZ-prep from Ventana ${ }^{\circledR}$ was used. Afterwards slides were pretreated with Ventana CC1 (cell conditioning pH8.5) for $64 \mathrm{~min}$. and incubated with antibody $32 \mathrm{~min} . / 36^{\circ}$ diluted $1: 100$ in Dako antibodydiluent S2022 In Ventana Benchmark Ultra. The reaction was visualized by using Ventana Ultra View DAB-kit. Afterwards the sections were counterstained with Ventana Haematoxylin for $8 \mathrm{~min}$.

Twenty hot spot areas (i.e. 20 areas within the tumor with a high count of immunoreactive tumor nuclei) were estimated and the mean percentage of Ki-67 cells calculated. An experienced neruroendocrine tumor pathologist with no knowledge of patient related prognostic information calculated the Ki-67 index.

To perform immunohistochemical idenfication of chromogranin A in tumor tissue we used Polyclonal Rabbit Anti-Human Chromogranin A (Dako Denmark A/S ${ }^{\circledR}$, Glostrup, Denmark, code A0430). To identify positivity of synaptophysin and CD56, Monoclonal Mouse Anti Human Synaptophysin (Clone Snp 88, BioGenex Laboratories Inc. ${ }^{\circledR}$, Fremont, U.S.A., code MU363) and Monoclonal Mouse Anti-Human CD56 (Clone 1B6, NovoCastra ${ }^{\circledR}$, Trichem, Skanderborg, Denmark, code NCL-CD56-1B6) were used, respectively.

\section{Statistical analysis}

Survival probability and PFS was estimated by the method of Kaplan and Meier, and significance was tested by the log-rank test using the IBM ${ }^{\circledR}$ SPSS ${ }^{\circledR}$ Statistics 20 software. A p value below 0.05 was considered significant.

\section{Results}

Topotecan was administered as second-line treatment in five patients $(23 \%), 13$ patients $(59 \%)$ received toptecan as third-line and four patients $(18 \%)$ as fourth-line therapy.

Patients received a median of 2 courses (range 1-6) of treatment.

All 22 patients were eligible for response evaluation. No patient achieved complete response (CR) or partial response (PR). Five patients (23\%) had stable disease (SD) and 17 (77\%) had progressive disease (PD). Eight patients had no evaluation scans, four of them had unequivocal clinical progression and four patients had early death related to disease progression. Consequently, the disease control rate (CR + PR $+\mathrm{SD}$ ) was $23 \%$. The overall median survival time (OS) of the 22 patients was 3.2 months (Figure 1). The me- dian progression free survival (PFS) time in all patients was 2.1 months (Figure 2). The one-year survival rate was $18 \%$. The five patients with SD after 3 courses had a significantly longer survival of 7.1 months versus 3.0 months in patients with $\mathrm{PD}(\mathrm{P}=$ $0.04)$.

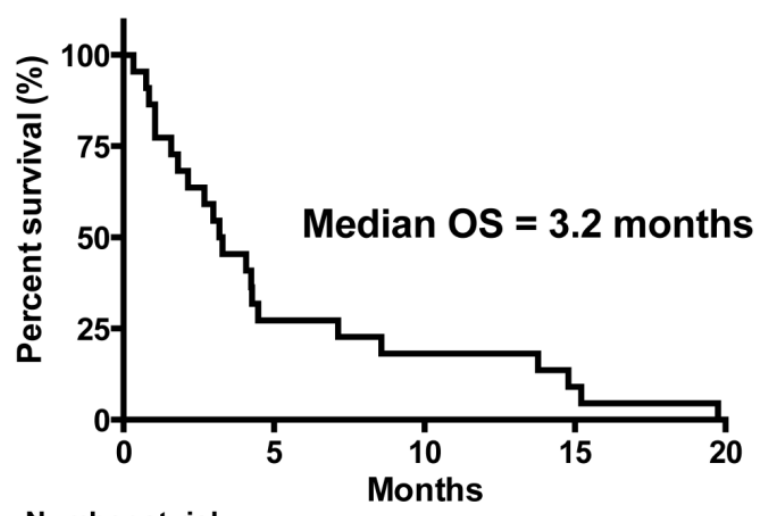

Number at risk

$\begin{array}{llll}22 & 6 & 4 & 2\end{array}$

Figure I. Overall Survival (OS) $(n=22)$.

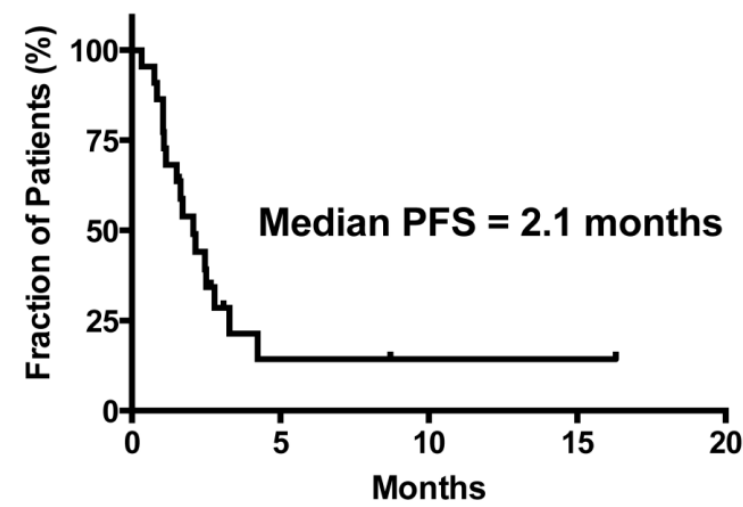

Number at risk

22

2

1

1

Figure 2. Progression Free Survival (PFS) $(n=22)$.

No statistically significant difference was identified in OS by subgroup analysis between patients who received topotecan as second-line chemotherapy $(\mathrm{n}=$ 5; OS 3.3 months), in patients who received topotecan as third-line therapy ( $\mathrm{n}=13$; OS 4.2 months) or in patients who received topotecan as fourth line therapy ( $\mathrm{n}=4$; OS 2.1 months) $(\mathrm{P}=0.30)$.

Patients in WHO PS 0-1 ( $\mathrm{n}=16)$ had an OS of 4.1 months versus 3.0 months in patients in WHO PS 2-4 $(\mathrm{n}=6)(\mathrm{P}=0.36)$.

Patients with a Ki-67 index $\geq 55 \%(n=17)$ had a similar PFS of 2.1 months compared to patients with Ki-67 index $<55 \%(\mathrm{n}=5$; PFS 2.5 months) $(\mathrm{P}=0.54)$.

By comparing OS of men $(n=11)$ and female $(n=$ 11), 4.3 months versus 2.1 months respectively, no statistical significant influence of sex on status of sur- 
vival was recorded $(\mathrm{P}=0.17)$

The treatment-related toxicities associated with topotecan in this patient population were relatively mild. Three patients (14\%) experienced CTC grade 3 leucopenia, two patients grade $4(9 \%)$ and three patients (14\%) grade 3 thrombocytopenia. Febrile neutropenia occured in one patient (5\%). Emesis was well controlled on the standard prophylactic antiemetic therapy, and no patients suffered from diarrhea. There were no treatment-related deaths.

\section{Discussion}

First-line treatment of NECs based on platin and etoposide is widely recommended [7-9]. There is no established second-line therapy for NECs. Recent studies have demonstrated the efficacy of temozolomide alone or in combination with capecitabine \pm bevacizumab. Particularly, there was a trend towards better survival in pancreatic NECs, in patients with a good performance status, and patients with Ki67<60\% [10,11].

In the NANETS and the NCCN guidelines, topotecan therapy is mentioned as a treatment option $[4,5]$. Nonetheless, actually the present study is the first to report on the efficacy of topotecan as monotherapy in heavily pretreated NEC patients. Usually these patients rapidly deteriorate after first-line treatment and have a short survival. In our study, none of the 22 patients obtained objective response $(\mathrm{CR}+\mathrm{PR})$ to treatment. However, it is noteworthy that the treatment resulted in a disease control rate of $23 \%$ and a one-year survival rate of $18 \%$ in patients with tumor progression at study entry.

The study population had a high median Ki67 index of $95 \%$ and exhibited responsiveness, although not radiological objective responses, to topotecan. Recent studies of temozolomide as second line therapy for NEC patients had mostly responders in patients with low Ki67 indices $(<50-55 \%)$ [10,11]. Recent data indicate that it might not be correct to consider all NEC as one single disease entity as in the present WHO classification (11) and that new treatments may be worth pursuing, guided by the Ki67 proliferative index and the site of tumor origin [12]. NEC patients with $\mathrm{Ki} 67>55 \%$ are probably more responsive to platin-based chemotherapy, but have a shorter survival than patients with a lower Ki67. This is not contradicted in the present study.

The Nordic Guidelines 2010 [1] describes IHC of NECs as synaptophysin positive but frequently negative for CgA. In our cohort one patient (5\%) stained negative for $\mathrm{CgA}$ another patient $(5 \%)$ stained negative for synaptophysin. Again, this reflects the heterogeneity of NECs wherefore other diagnostic markers are warranted.
The treatment strategy in advanced NEC remains a clinical challenge because of the lack of effective options and the absence of well-designed controlled randomized clinical trial data to support evidence-based practice. The majority of the studies reported so far vary exceedingly in terms of patient selection and type of chemotherapy.

The efficacy and toxicity results of topotecan as monotherapy in G3 NEC are quite similar to those in the second line treatment of small cell cancer, in which the treatment is one of the few treatment options [13].

Topotecan monotherapy shows modest anti-tumor activity in heavily treated patients with progressive disseminated G3 NECs. And as the toxicity of topotecan is tolerable, further studies of topotecan-based regimens are warranted.

\section{Article information}

\section{Contributors}

SWL initiated the study. Study design and analytical strategy were developed by all authors. IHO made the data management supervised by SWL, UK, $\mathrm{AK}$ and BF. IHO wrote the first version of the manuscript and all authors took part in the revision. All authors have seen and approved the final version of this paper.

\section{Funding}

This research received no specific grant from any funding. Agency in the public, commercial or not-for-profit sectors.

\section{Provenance and peer review}

Not commissioned; externally peer reviewed.

\section{Data sharing statement}

There are no additional data available.

\section{Competing Interests}

\author{
None.
}

\section{References}

1. Janson ET, Sorbye H, Welin S, Federspiel B, Grønbaek H, Hellman P, et al.: Nordic Guidelines 2010 for diagnosis and treatment of gastroenteropancreatic neuroendocrine tumours. Acta Oncol 2010 Aug:49:740-756.

2. Moertel CG, Kvols LK, O'Connell MJ, Rubin J: Treatment of neuroendocrine carcinomas with combined etoposide and cisplatin. Evidence of major therapeutic activity in the anaplastic variants of these neoplasms. Cancer $1991 \mathrm{Jul}$ $14 ; 68: 227-232$.

3. O'Brien MER, Ciuleanu T-E, Tsekov H, Shparyk Y, Cuceviá B, Juhasz G, et al.: Phase III trial comparing supportive care alone with supportive care with oral topotecan in patients with relapsed small-cell lung cancer. J Clin Oncol 2006 Nov 30;24:5441-5447.

4. Strosberg JR, Coppola D, Klimstra DS, Phan AT, Kulke MH, Wiseman GA, et al.: The NANETS Consensus Guidelines for the Diagnosis and Management of Poorly Differentiated (High-Grade) Extrapulmonary Neuroendocrine Carcinomas. Pancreas 2010 Aug;39:799-800.

5. CML Gastro 31.4 LA Langer 2012: 1-6.

6. 2012ESMOHandbookClinPharmacolAntiCancerAgents 2012: 1-226. 
7. Fjällskog M, Granberg D, Welin S, Eriksson C: Treatment with cisplatin and etoposide in patients with neuroendocrine tumors. Cancer 2001.

8. Olsen IH, Langer SW, Jepsen I, Assens M, Federspiel B, Hasselby JP, et al.:

First-line treatment of patients with disseminated poorly differentiated neuroendocrine carcinomas with carboplatin, etoposide, and vincristine: A single institution experience. Acta Oncol 2012 Jan;51:97-100.

9. Yao J.: One hundred years after "carcinoid": epidemiology of and prognostic factors for neuroendocrine tumors in 35,825 cases in the United States. J Clin Oncol 2008 Jan 20;26:3063-3072.

10. Olsen IH, Sørensen JB, Federspiel B, Kjær A, Hansen CP, Knigge U, et al.: Temozolomide as Second or Third Line Treatment of Patients with Neuroendocrine Carcinomas. The Scientific World Journal 2012;2012:1-4.

11. Welin S, Sorbye H, Sebjornsen S, Knappskog S, Busch C, Oberg K: Clinical effect of temozolomide-based chemotherapy in poorly differentiated endocrine carcinoma after progression on first-line chemotherapy. Cancer 2011 Oct 15;117:4617-4622.

12. Sorbye $\mathrm{H}$, Welin $\mathrm{S}$, Langer SW, Vestermark LW, Holt N, Osterlund $\mathrm{P}$, et al.: Predictive and prognostic factors for treatment and survival in 305 patients with advanced gastrointestinal neuroendocrine carcinoma (WHO G3): The NORDIC NEC study. Annals of Oncology 2012 Dec 18;24:152-160.

13. Garst J: Topotecan: An evolving option in the treatment of relapsed small cell lung cancer. Ther Clin Risk Manag 2007 Dec;3:1087-1095. 